\title{
The Story of Dark Matter
}

\author{
Brian Albert Robson \\ Additional information is available at the end of the chapter \\ http://dx.doi.org/10.5772/intechopen.75662
}

\begin{abstract}
Several astronomical observations concerning the structure of galaxies, the rotation of stars in spiral galaxies, the motions of galaxies within a cluster of galaxies, and so on, cannot be understood in terms of Newton's universal law of gravitation and the visible atomic matter within the galactic systems. This chapter reviews the progress made over many decades in the understanding of these cosmological observations that indicate a serious breakdown of Newton's universal law of gravitation unless there exists additional unseen matter, named "dark matter." The only alternative to "dark matter" is to modify Newtonian gravity. The chapter presents a critical review of the two main approaches to providing the additional gravity required to understand the puzzling astronomical observations: (1) the "dark matter" hypothesis providing additional unseen mass and (2) modification of Newton's universal law of gravity such that there is a stronger gravitational field at larger distances. Both Milgrom's modified Newtonian dynamics (MOND) theory and Robson's recent quantum theory of gravity provided by the generation model (GM) of particle physics are discussed.
\end{abstract}

Keywords: gravity, dark matter, MOND theory, generation model

\section{Introduction}

The notion of "dark matter" emerged from several astronomical observations concerning the structure of galaxies, the rotation of stars and neutral hydrogen gas in spiral galaxies, the motions of clusters of galaxies, and so on. These observations could not be described in terms of Newton's universal law of gravitation and the visible ordinary atomic matter within the galactic systems. This chapter reviews the progress made over many decades in the understanding of these cosmological observations that indicated a serious breakdown of Newton's universal law of gravitation unless there existed additional unseen matter that was named "dark matter." The only alternative to "dark matter" was to modify Newtonian gravity. 
This chapter presents a critical review of the two main approaches to providing the additional gravity required to understand the puzzling astronomical observations: (1) the "dark matter" hypothesis providing additional unseen mass and (2) modification of Newton's universal law of gravity such that there is a stronger gravitational field at larger distances. Both Milgrom's modified Newtonian dynamics (MOND) theory and Robson's recent quantum theory of gravity provided by the generation model (GM) of particle physics are discussed.

\section{The notion of dark matter}

The notion of "dark matter" emerged from observations of large astronomical objects such as galaxies and clusters of galaxies, which displayed gravitational effects that could not be accounted for by the visible matter: stars, gas, and so on, assuming the validity of Newton's universal law of gravitation.

It was concluded that such observations could only be described satisfactorily if there existed stronger gravitational fields than those provided by the visible matter and Newtonian gravity. Such gravitational fields required either more mass or an appropriate modification of Newton's universal law of gravitation.

Early preliminary evidence for such a "mass discrepancy" was observed in 1933 by Zwicky [1] for the Coma cluster of galaxies. He estimated that the cluster contained considerably more "dark matter" than the visible galactic matter in order to account for the fast motions of the galaxies within the cluster and also to hold the cluster together.

Additional preliminary evidence for the mass discrepancy was found by Babcock [2] in 1939 and Rubin and Ford [3] in 1970 by measuring the rotation curve of the Andromeda galaxy, the nearest spiral galaxy to the Milky Way. The rotation curve of a galaxy is the dependence of the orbital velocity of the visible matter in the galaxy on its radial distance from the center of the galaxy. However, neither Babcock nor Rubin and Ford attributed their observations of an increase in mass toward the edge of the galaxy to any missing mass.

In 1970, Freeman [4] found rotation curves for several galaxies that disagreed with expectation based upon the assumption that the galaxies consisted of stars, gas, and nothing else. Freeman suggested that these galaxies, like the Coma cluster observed much earlier by Zwicky, contained considerably more invisible "dark matter" than the luminous matter. In 1973, Roberts and Rots [5], using 21-cm line data, obtained neutral hydrogen rotation curves of three nearby spiral galaxies. These rotation curves extended to considerably larger distances from the centers of the galaxies than the corresponding rotation curves for the stars. In each case, the complete rotation curve was essentially "flat" out to the edge of the $21-\mathrm{cm}$ data.

In 1974, Ostriker et al. [6] stated that the current observed rotation curves strongly indicated that the mass of a spiral galaxy increases approximately linearly with radius to about $1 \mathrm{Mpc}$ so that the ratio of the total mass to the observed visible mass was large. They concluded that the rotation curves could most plausibly be understood if the spiral galaxy was embedded in a giant spherical halo of invisible "dark matter." 
Further evidence for the dark matter hypothesis in many spiral galaxies was obtained in the 1970s by Rubin et al. [7], who measured high-quality optical rotation curves for the luminous matter and Bosma [8], who compiled 21-cm rotation curves for the neutral hydrogen gas that extended far beyond the luminous matter of each galaxy. In all these cases, the complete rotation curve was essentially "flat" out to the edge of the $21-\mathrm{cm}$ data.

By 1980, the conclusive observation from the rotation curves of spiral galaxies was that there existed a major "mass discrepancy" that was greater if larger distance scales were involved. This implied that if Newton's universal law of gravitation was approximately valid, as in the Solar System, considerably more mass was required to be present in each galaxy. This invisible matter was termed "dark matter" with the introduction of the dark matter hypothesis: each spiral galaxy was embedded in a huge spherical halo of dark matter.

Thus, the notion of "dark matter" essentially emerged from the observed rotation curves of spiral galaxies that provided convincing evidence for a mass discrepancy within the galaxy. The only alternative to "dark matter" seemed to be a significant modification of Newton's universal law of gravitation to provide the required stronger gravitational field at larger distance scales. However, at that time, such a modification of Newtonian gravity was not considered a viable alternative.

\section{The dark matter hypothesis}

The dark matter hypothesis was essentially established in 1974 by Ostriker et al. [6], who concluded that the rotation curves of spiral galaxies could most plausibly be understood if the spiral galaxy was embedded in a giant spherical halo of invisible "dark matter." In the conventional cosmological model of spiral galaxies [9], each spiral galaxy is considered to be surrounded by a giant halo of invisible (dark) matter that provides a large contribution to the gravitational field at large distances from the center of the galaxy.

Standard model of cosmology [10] assumes that the universe is now composed of about 5\% ordinary matter, $27 \%$ dark matter, and $68 \%$ dark energy, so that dark matter constitutes about $84 \%$ of the total mass, while dark energy plus dark matter constitute about $95 \%$ of the total mass-energy content of the universe. Thus, for many years, cosmologists have been confronted with the notion that $84 \%$ of the gravitational mass of the universe is dark matter.

The hypothesis of a dark matter spherical halo surrounding a spiral galaxy to account for the observed flat rotation curve of the galaxy has yet to be verified. One of the main difficulties is that the nature of the proposed dark matter is unknown.

Initially, massive compact halo objects (MACHOs), were searched for within the outer regions of galaxies, using microlensing techniques [11]. The conclusion from these observations was that at most $20 \%$ of a galactic halo consists of MACHOs, and the rest of the halo consists of nonbaryonic matter.

The only other known candidates for dark matter are the three neutrinos of the standard model (SM) of particle physics [12]. However, it was demonstrated in 1983 [13] that if dark 
matter consisted entirely of neutrinos, the large-scale structure of the universe would significantly differ from the observed one, since the neutrinos are relativistic particles leading to a smooth large-scale structure. Recently, Frampton [14] has suggested that the nonbaryonic component of dark matter may consist entirely of primordial intermediate mass black holes. However, this suggestion remains to be verified.

The existence of dark matter in the universe suggests that one requires new physics beyond the SM. Three such particles have been searched for without success: (1) axions, (2) weakly interacting massive particles (WIMPS), and (3) sterile neutrinos. These three particles are all hypothetical particles, some of which have been introduced into particle physics in order to resolve certain perceived problems.

The axion was postulated in 1977 by Peccei and Quinn [15] in an attempt to understand the strong $\mathrm{CP}$ problem in quantum chromodynamics (QCD). To date various experiments have been carried out but none have successfully identified an axion particle.

A weakly interacting massive particle (WIMP) is considered to be a new elementary particle, which only interacts via gravity and any other weak force. The basic goal of direct detection of a (WIMP) is to measure the energy deposited when it interacts with nuclei in a detector, transferring energy to nuclei. Such direct-detection experiments need to be carried out deep underground to prevent them being swamped by unwanted noise from cosmic ray particles.

The most favored (WIMP) is the lightest neutral stable particle, the neutralino, predicted by the supersymmetric (SUSY) theory of particle physics, which provides a significant relationship between elementary bosons and fermions. This relationship resolves several puzzling problems, including the hierarchy problem, for example, the extremely large difference in the strengths of the gravitational and weak interactions $\approx 10^{-36}$. However, to date, no evidence for any SUSY particle has been found either at the large Hadron collider (LHC) in CERN or in the many underground detection laboratories. At the LHC, no previously unknown particles, which may be evidence of SUSY, have been observed since the claimed detection of the Higgs boson, so that SUSY probably does not exist. In addition, no (WIMP) has clearly been detected over several decades at any of the underground laboratories such as the large underground xenon (LUX) experiment in the Homestake Mine, Dakota.

However, there has been one claim of direct detection of dark matter from the DAMA-LIBRA experiment at the Gran Sasso laboratory [16]. This experiment has observed a possible dark matter event rate that modulates annually as the Earth travels around the Sun, while the Solar System moves within the disk of the Milky Way and hence through the hypothesized galactic dark matter halo. The count rate is expected to depend upon the relative velocity of the detector and undergoes a modulation that peaks in June, when the relative velocity is at its maximum.

This observation of the DAMA-LIBRA experiment is controversial, since it has been excluded by observations from several direct-detection experiments, including perhaps the most sensitive one, the LUX experiment. In order to test the DAMA-LIBRA claim, a more sensitive directdetection experiment, SABRE, is being undertaken with improved but similar equipment in Australia [17]. 
Sterile neutrinos are also hypothetical neutral particles that emerged from the development of the electroweak theory by Glashow [18, 19], who separated the neutrinos into left-handed and right-handed particles. The left-handed neutrinos interact via the left-handed weak interaction, while the right-handed neutrinos do not and only interact via gravity. The right-handed neutrinos correspond to the so-called sterile neutrinos.

The possible existence of sterile neutrinos arose in the development of the SM at a time when the neutrinos were considered to be massless. This is no longer the case so that the three "normal" neutrinos are expected to have right-handed components with the same mass as the left-handed components and hence are unsuitable as candidates for dark matter.

\section{Milgrom MOND theory}

In view of the considerable uncertainties concerning the existence and nature of the proposed dark matter, there have been several attempts to modify Newton's universal law of gravitation instead of introducing dark matter.

In 1983, Milgrom [20] developed a modification of Newtonian dynamics known as the MOND theory, as a possible alternative to dark matter. This theory is based upon describing two astronomical observations: (1) the flat rotational curves of spiral galaxies at large distances from their centers and (2) the Tully-Fisher empirical relation [21], which states that the intrinsic luminosity $L$ (proportional to the total visible mass) of a spiral galaxy and the velocity, $v_{f}$, of the matter circulating at the extremities of the galactic disks are given by:

$$
L \propto v_{f}^{\alpha}
$$

where $\alpha$ is approximately 4 .

In order to describe both the flat rotation curves of spiral galaxies and the Tully-Fisher relation, Milgrom suggested that gravity varies from the prediction of Newtonian dynamics for low accelerations. In particular, the transition from $1 / r^{2}$ to $1 / r$ gravity should occur below a critical "acceleration" $a_{0}$ rather than beyond a critical distance $r_{0}$ : the former leads to the Tully-Fisher relation, while the latter leads to:

$$
L \propto v_{f}^{2}
$$

in gross disagreement with the Tully-Fisher relation.

The modified law of gravity in terms of $a_{0}$ is [22]:

$$
g=G M / r^{2}+\left(G M a_{0}\right)^{\frac{1}{2}} / r
$$

where the first term corresponds to distances for which the acceleration is $\gg a_{0}$ and the second term corresponds to distances associated with the flat rotation curves, that is, with $v_{f}$. Indeed the second term gives: 


$$
v_{f}=\left(G M a_{0}\right)^{\frac{1}{4}}
$$

which, if the mass to luminosity ratio, $M / L$, is roughly constant for galaxies, leads to the TullyFisher relation.

To summarize: MOND is an empirical modification of Newton's gravitational interaction that is designed to provide agreement with two overarching observational facts: (1) the flat rotation curves of spiral galaxies and (2) the Tully-Fisher relation. It achieves this aim by causing the gravitational interaction to change from $1 / r^{2}$ for small distances, $r$, to $1 / r$ at large galactic distances as the gravitational acceleration becomes less than a critical small acceleration $a_{0} \approx 1.2 \times 10^{-10} \mathrm{~m} \mathrm{~s}^{-2}$.

However, MOND is incomplete in the sense that in order to be more acceptable to the overall scientific community, it needs to be related to a more general underlying theory of gravity. Just as Kepler's laws of planetary motion described mathematically but without any physical content the observed orbits of the planets, it required Newton's universal law of gravitation to understand the physics underlying Kepler's laws.

\section{Robson quantum theory of gravity}

The generation model (GM) [23] of particle physics has been developed over many years as a viable alternative to the standard model (SM) [12] of particle physics. The SM is considered by the majority of physicists to be incomplete in the sense that it provides no understanding of many empirical observations including the existence of three families or generations of leptons and quarks, which apart from mass have similar properties, a nonunified description of the origin of mass, and the nature of the gravitational interaction.

The GM overcomes the incompleteness inherent in the SM by introducing three important different assumptions [24]: (1) a simplified unified classification scheme of the leptons and quarks in terms of additive quantum numbers, (2) an alternative version of quark mixing for hadronic processes, and (iii) the weak interactions are not fundamental interactions.

The development of the GM, primarily to describe the three generations of leptons and quarks of the SM [25], employing a unified classification scheme involving only three conserved additive quantum numbers, led to a composite model of the leptons and quarks and also the weak bosons, $W$ and $Z$, mediating the weak interactions [23, 24].

Thus, the essential difference between the GM and the SM is that in the GM the leptons, quarks, and weak bosons are composite particles rather than elementary particles as in the SM.

In the GM, the leptons, quarks, and weak bosons consist of massless spin-1/2 particles called rishons and/or their antiparticles (antirishons). Each rishon carries a single color charge-red, green or blue-and each antirishon carries an anticolor charge-antired, antigreen or antiblue.

The first generation of leptons and quarks comprising the electron, the electron neutrino, the up quark, and the down quark are composed of two kinds of rishons: a T-rishon with electric charge $Q=+\frac{1}{3}$ and a $V$-rishon with $Q=0$ and/or their antiparticles: a $\bar{T}$-antirishon with 
electric charge $Q=-\frac{1}{3}$ and a $\bar{V}$-antirishon with $Q=0$. Both the $T$-rishon and the $V$-rishon were introduced in 1979 by Harari [26] in his schematic model of the first generation of leptons and quarks describing their electric charge states.

The second and third generations of leptons and quarks are composed of the same "core" rishons and/or antirishons as the first generation plus the addition of one and two rishonantirishon pair(s), $\Pi$, respectively, where

$$
\Pi=[(\bar{U} V)+(\bar{V} U)] / \sqrt{2}
$$

and the $U$-rishon has $Q=0$ and carries a single color charge [23].

The constituents of the leptons and quarks are bound together by a strong QCD color-type interaction [27], corresponding to a local gauged SU(3) field (analogous to QCD in the SM) mediated by massless hypergluons (analogous to gluons in the SM).

The nature of the hypergluon fields acting between the rishons and/or antirishons of the composite leptons and quarks are analogous to the gluon fields acting between quarks and/or antiquarks in the SM. In particular, the nature of the hypergluon fields is such that they lead to a runaway growth of the fields surrounding an isolated color charge, implying that an isolated rishon or antirishon would have an infinite energy associated with it [28]. Nature requires such infinities to be essentially canceled or at least made finite. It does this for the composite systems of rishons and/or antirishons by requiring that the composite particle be colorless. However, quantum mechanics prevents these color charges from occupying exactly the same place so that the color fields are not exactly canceled although sufficiently to remove the infinities associated with isolated rishons or antirishons.

In the GM, each lepton of the first generation is colorless being composed of three antirishons carrying different anticolors. On the other hand, each quark of the first generation is colored being composed of one rishon and one colorless rishon-antirishon pair [23].

The second and third generations are identical to the first generation plus one and two colorless rishon-antirishon pairs, respectively, so that all leptons are colorless and all quarks are colored. Consequently, leptons do not combine to form more complex systems, while the quarks form hadrons that consist of two families: colorless baryons, made of three quarks with different color charges, and colorless mesons, made of one quark and one antiquark with opposite color charges [23].

Within the framework of the GM, the assumption that the elementary particles of the SM-the six leptons, six quarks, and three weak bosons-are all composite particles has led to a unified origin of mass [29] and a quantum theory of gravity [30].

In 1905, Einstein concluded [31] that the mass of a body $m$ is a measure of its energy content $E$ and is given by $m=E / c^{2}$, where $c$ is the speed of light in a vacuum. Recently, this relationship has been verified [32] to within $0.00004 \%$ for atomic systems.

In the SM, the mass of a hadron arises mainly from the energy content of its constituent quarks and gluons, in agreement with Einstein's conclusion. However, the masses of the elementary particles - the leptons, quarks, and weak bosons - are interpreted [33] in a completely different 
way involving a Higgs field [34, 35]. Thus, the SM does not provide a unified origin of mass, contrary to Einstein's conclusion. Furthermore, the so-called Higgs mechanism does not provide any physical explanation for the origin of the masses of the leptons, quarks, and weak bosons, as pointed out by Lyre [36].

In the GM, the elementary particles of the SM are composite particles. Since the mass of a hadron originates mainly from the energy of its constituents, the GM postulates that the mass of a lepton, quark, or weak boson arises from a characteristic energy associated with its constituent massless rishons, antirishons, and hypergluons. The mass of each of these composite particles arises from the energy stored in the motion of the rishons and/or antirishons and the energy of the color hypergluon fields, $E$, according to Einstein's equation $m=E / c^{2}$. Thus, unlike the SM, the GM provides a unified description of the origin of all mass and hence has no requirement for a Higgs field to generate the mass of any particle.

Since, to date, there is no direct evidence for any substructure of leptons or quarks, it is expected that the rishons and/or antirishons of each lepton or quark are localized within a very small volume of space by the strong "intrafermion" color interactions, acting between the colored rishons and/or antirishons.

In the GM, the mass hierarchy of the three generations arises from the substructures of the leptons and quarks. The mass of a composite particle will be greater if the degree of localization of its constituents is smaller, as a consequence of the nature of the strong intrafermion color interactions possessing the property of asymptotic freedom [37, 38], whereby the color interactions become stronger for larger separations of the color charges, as a result of antiscreening effects. In addition, particles with two or more like electrically charged rishons or antirishons will have larger structures due to electric repulsion. Ref. [23] presents a qualitative understanding of the mass hierarchy of the three generations of leptons and quarks: a quantitative calculation of the mass hierarchy requires very sophisticated computations.

On the other hand, the SM, involving the Higgs field to generate the masses of its elementary leptons and quarks, is unable to provide any understanding of the mass hierarchy of the three generations. As Lyre [36] has pointed out, the introduction of the Higgs field into the SM simply corresponds mathematically to putting in "by hand" the masses of the elementary particles of the SM.

The GM also provides a quantum theory of gravity. Gravitational interaction acts between particles with mass. Such particles are composed of rishons and/or antirishons that carry colored or anticolored charges and hence are required to be colorless in order to avoid infinite energies within their systems.

In the GM, the constituent electrons, neutrons, and protons of ordinary matter are all composite and colorless fermion particles. Between any two such fermion particles, there exists a residual interaction arising from the color interactions acting between the rishons and/or antirishons of one fermion and the color-charged constituents of the other fermion. Robson proposed $[29,30]$ that such "interfermion" color interactions could be identified with the usual gravitational interaction.

In the GM, gravity essentially emerges from the residual color forces between all electrons, neutrons, and protons. This leads $[22,39]$ to a new law of gravity: the residual color interactions 
between any two bodies of masses $m_{1}$ and $m_{2}$, separated by a distance $r$, lead to a universal law of gravitation, which closely resembles Newton's original law given by:

$$
F=H(r) m_{1} m_{2} / r^{2}
$$

where Newton's gravitational constant is replaced by a function of $r, H(r)$.

Both the fundamental intrafermion and the residual interfermion color interactions possess two properties arising from the self-interactions of the hypergluons mediating these interactions: (1) asymptotic freedom and (2) color confinement [39].

The antiscreening effects arising from the self-interactions of the hypergluons cause the color interactions to become stronger for larger separations of the color charges. In the case of the fundamental intrafermion interactions, this results in an increase in the characteristic energy and hence the mass of a composite particle that is less localized, as discussed earlier. In the case of the residual interfermion (gravitational) interactions acting between two masses, it leads to an increase in the strength of the gravitational interaction for larger separations so that $H(r)$ becomes an increasing function of $r$.

It is known from particle physics that the strong color interactions tend to increase with the separation of color charges, and for large separations, this increase is approximately a linear function of $r$ [40], in agreement with the flat rotation curves observed for spiral galaxies. Thus, $H(r)$ is expected to be approximately a linear function of $r$ :

$$
H(r)=G\left(1+k r / r_{S}\right)
$$

where G is Newton's gravitational constant, $k$ represents the relative strengths of the modified and Newtonian gravitational fields, and $r_{S}$ is a radial length scale dependent upon the radial mass distribution of the spiral galaxy so that $r_{S}$ varies from galaxy to galaxy.

Thus, the modified law of gravity in the GM may be written as:

$$
g=G M / r^{2}+G M k /\left(r r_{S}\right) .
$$

Eq. (8) is very similar to Eq. (3) of the MOND theory and one can relate the modified terms in the two gravitational acceleration expressions to obtain:

$$
a_{0}=G M\left(k / r_{S}\right)^{2}
$$

Thus, the scale length $r_{S}$ may be regarded as the radial parameter beyond which weak acceleration takes place. Eq. (9) implies that the physical basis of the critical weak acceleration $a_{0}$ of the MOND theory is the existence of a radial parameter $r_{S}$ that defines a region beyond which the gravitational field behaves essentially as $1 / r$.

To summarize: gravity in the GM is identified with the very weak, universal, and attractive residual color interactions acting between the particles of ordinary matter. This gravitational interaction is mediated by hypergluons, which self-interact, leading to a significant modification of Newton's universal law of gravitation, especially at galactic distance scales. However, 
the self-interactions of the hypergluons cease at a sufficiently large distance as a consequence of the color confinement property associated with the QCD-like gravitational interaction. This leads to a finite range of the gravitational interaction for very large cosmological distances, estimated to be $\approx$ several billion light years [39].

Eq. (8) describes both the flat rotation curves of spiral galaxies and also the Tully-Fisher relation [22]. This modification of Newton's universal law of gravitation is essentially equivalent to that of the MOND theory, in that both describe these two overarching observational facts. However, the GM is based upon a quantum field theory of gravity, which provides a general underlying theory of gravity and hence a more physical understanding of the MOND results. Furthermore, unlike the MOND theory, the quantum theory of gravity provides a possible understanding of the observed "accelerating" expansion of the universe [41, 42].

\section{Conclusion and discussion}

In 1980, the conclusive observation from the flat rotation curves of spiral galaxies was that there existed a significant "mass discrepancy" in spiral galaxies, which was greater if larger distance scales were involved. The flat rotation curves indicated that either Newton's universal law of gravitation and hence the General Theory of Relativity [43] required modification at galactic distances or significantly more mass than the visible mass was required to be present within each galaxy.

The possible mass discrepancy within a galaxy led to the dark matter hypothesis whereby each spiral galaxy is embedded within a huge spherical halo of dark matter. The only alternative to dark matter was considered to be an appropriate modification of Newtonian gravity to provide the required extra gravitational field at large (galactic) distances.

Initially, the dark matter hypothesis was favored, since, at that time, a significant modification of Newtonian gravity was not considered a viable alternative. However, the dark matter hypothesis has several problems: (1) the nature of the proposed dark matter is unknown, although it is considered to be mainly nonbaryonic matter, (2) a dark matter halo has not yet been detected directly, (3) the density profile of a typical halo is required to be fine-tuned in order to produce the observed flat rotation curve of a spiral galaxy, and (4) the lack of dark matter in large globular clusters, which have about the same mass as the smallest dwarf galaxies that are considered to have considerable amounts of dark matter, is a mystery.

Several hypothetical particles have been suggested for the nonbaryonic component of dark matter but, to date, no clear evidence for the existence of any of these particles (axions, WIMPS, or sterile neutrinos) has been obtained.

More recently, modified gravity theories such as the MOND theory have gained popularity, since they overcome most of the problems associated with the dark matter hypothesis. In particular, Mond theory describes the flat rotation curves of spiral galaxies without finetuning, and the globular clusters' lack of dark matter is expected to arise from their much smaller size relative to a dwarf galaxy. 
The gravitational interaction of the GM, identified with the very weak, universal, and attractive residual $Q C D$ color interactions acting between ordinary matter particles, is essentially equivalent to that of the MOND theory, in that both describe successfully the flat rotation curves of spiral galaxies and the Tully-Fisher relation. However, the GM quantum theory of gravity, based upon a quantum field theory, provides not only a general underlying theory of gravity and hence a more physical understanding of the MOND theory but also a possible understanding of the so-called dark energy causing the observed accelerating expansion of the universe. Indeed, the GM quantum theory describes both dark matter and dark energy in terms of two intrinsic properties of the residual QCD color interactions: antiscreening at galactic distances and finite range at cosmological distances.

The continuing success $[44,45]$ of the MOND theory together with the underlying GM quantum field theory of gravity is a strong argument against the existence of undetected dark matter halos consisting of unknown matter embedding galaxies.

However, a direct empirical proof of the existence of dark matter is claimed to be provided by two colliding galaxies known as the "bullet cluster" [46]. Observations of the bullet cluster indicate that during the merging process, the dark matter, deduced from gravitational lensing, has passed through the collision point, while the baryonic component of matter, deduced from X-ray emission, has slowed down due to friction and has coalesced within a central region of the combined cluster. This separation of the two kinds of matter is claimed to provide evidence for dark matter. Unfortunately, a similar separation of the regions of non-Newtonian gravity in the MOND and GM gravity theories are expected to occur in the merging of two galaxies such as the bullet cluster, so that these modified gravity theories also describe the merging of the bullet cluster.

\section{Author details}

Brian Albert Robson

Address all correspondence to: brian.robson@anu.edu.au

The Australian National University, Canberra, ACT, Australia

\section{References}

[1] Zwicky F, Die Rotverschiebung von extragalaktischen Nebeln. Helvetica Physica Acta. 1933;6:110-127

[2] Babcock HW. The rotation of the Andromeda nebula. Lick Observatory Bulletin. 1939;19: $41-51$

[3] Rubin VC, Ford WK. Rotation of the Andromeda nebula from a spectroscopic survey of emission regions. The Astrophysical Journal. 1970;159:379-403 
[4] Freeman KC. On the disks of spiral and S0 galaxies. The Astrophysical Journal. 1970;160: 811-830

[5] Roberts MS, Rots AH. Comparison of rotation curves of different galaxy types. Astronomy and Astrophysics. 1973;26:483-485

[6] Ostriker JP, Peebles PJE, Yahil A. The size and masses of galaxies and the mass of the universe. The Astrophysical Journal. 1974;193:L1-L4

[7] Rubin VC, Ford KW, Thonnard N. Rotational properties of 21 Sc galaxies with a large range of luminosities and radii, from NGC 4605 ( $R=4 \mathrm{kpc}$ ) to UGC 2885 ( $R=122 \mathrm{kpc}$ ). The Astrophysical Journal. 1980;238:471-487

[8] Bosma A. The distribution and kinematics of neutral hydrogen in spiral galaxies of various morphological types. Groningen University; $1978 ; \mathrm{PhD}$ thesis

[9] Ostriker JP, Steinhardt PJ. The observational case for a low-density universe with a nonzero cosmological constant. Nature. 1995;377:600-602

[10] Ade PAR et al. (Planck Collaboration). Planck 2013 results. I Overview of products and scientific results. Astronomy and Astrophysics. 2014;571 Article ID A1, 48pp

[11] Alcock $\mathrm{C}$ et al. The MACHO project: Microlensing results from 5.7 years of large magellanic cloud observations. Astrophysical Journal. 2000;542:281-307

[12] Gottfried K, Weisskopf VF. Concepts of Particle Physics. Vol. 1. New York: Oxford University Press; 1984. 189p

[13] White SDM, Frenk CS, Davis M. Clustering in a neutrino-dominated universe. Astrophysical Journal. 1983;274:L1-L5

[14] Frampton PH. Theory of dark matter. In: Fritzsch H, editor. Cosmology, Gravitational Waves and Particles. Singapore: World Scientific; 2018. pp. 76-89

[15] Peccei RD, Quinn HR. Constraints imposed by CP conservation in presence of pseudoparticles. Physical Review D. 1977;16:1791-1797

[16] Belli P et al. Observations of annual modulation in direct detection of relic particles and light neutralinos. Physical Review. 2011;84. Article ID 055014 13pp

[17] Froberg F. SABRE: WIMP modulation detection in the northern and southern hemisphere. ArXiv: 1601.05307v1 [physics.ins.det]. 2016. 5pp

[18] Glashow SL. Partial symmetries of weak interactions. Nuclear Physics. 1961;22:579-588

[19] Robson BA. The generation model and the electroweak connection. International Journal of Modern Physics E. 2008;17:1015-1030

[20] Milgrom M. A modification of the newtonian dynamics as a possible alternative to the hidden mass hypothesis. Astrophysical Journal. 1983;270:365-370

[21] Tully RB, Fisher JR. New method of determining distances to galaxies. Astronomy and Astrophysics. 1977;54:661-673 
[22] Robson BA. The generation model of particle physics and galactic dark matter. International Journal of Modern Physics E. 2013;22. Article ID 1350067:11pp

[23] Robson BA. The generation model of particle physics. In: Kennedy E, editor. Particle Physics. Rijeka: InTech; 2012. pp. 1-28

[24] Robson BA. Progressing beyond the standard model. Advances in High Energy Physics. 2013;2013. Article ID 341738:12pp

[25] Veltman M. Facts and Mysteries in Elementary Particle Physics. Singapore: World Scientific; 2003. 340p

[26] Harari H. A schematic model of quarks and leptons. Physics Letters B. 1979;86:83-86

[27] Halzen F, Martin AD. Quarks and Leptons: An Introductory Course in Modern Particle Physics. New York: Wiley; 1984. 396p

[28] Wilczek F. In search of symmetry lost. Nature. 2005;433:239-247

[29] Robson BA. The generation model and the origin of mass. International Journal of Modern Physics E. 2009;18:1773-1780

[30] Robson BA. A quantum theory of gravity based on a composite model of leptons and quarks. International Journal of Modern Physics E. 2011;20:733-745

[31] Einstein A. Ist die Trägheit eines Körpers von seinem Energieinhalt abhängig. Annalen der Physik. 1905;18:639-641

[32] Rainville $S$ et al. World year of physics: a direct test of $E=m c^{2}$. Nature. 2005;438:10961097

[33] Aitchison IJR, Hey AJG. Gauge Theories in Particle Physics. Bristol: Hilger; 1982. 341p

[34] Englert F, Brout R. Broken symmetry and the mass of gauge vector bosons. Physical Review Letters. 1964;13:321-323

[35] Higgs PW. Broken symmetries and the masses of gauge bosons. Physical Review Letters. 1964;13:508-509

[36] Lyre H. Does the Higgs mechanism exist? International Studies in the Philosophy of Science. 2008;22:119-133

[37] Gross DJ, Wilczek F. Ultraviolet behavior of non-abelian gauge theories. Physical Review Letters. 1973;30:1343-1346

[38] Politzer HD. Reliable perturbative results for strong interactions. Physical Review letters. 1973;30:1346-1349

[39] Robson BA. Dark matter, dark energy and gravity. International Journal of Modern Physics E. 2015;24 Article ID 1550012:10pp

[40] Sumino Y. QCD potential as a "Coulomb-plus-linear" potential. ArXiv: 0303120v3 [hepph]. 2003:11pp 
[41] Riess AG et al. Observational evidence from supernovae for an accelerating universe and a cosmological constant. Astronomical Journal. 1998;116:1009-1038

[42] Perlmutter S et al. Measurements of omega and lambda from 42 high-redshift supernovae. Astrophysical Journal. 1999;517:565-586

[43] Einstein A. The basics of general relativity theory. Annalen der Physik. 1916;49:769-822

[44] McGaugh SS, de Blok WJG. Testing the hypothesis of modified dynamics with low surface brightness galaxies and other evidence. Astrophysical Journal. 1998;499:66-81

[45] Famaey B, McGaugh SS. Modified newtonian dynamics (MOND): Observational phenomenology and relativistic extensions. Living Reviews in Relativity. 2012;15. Article ID $10,159 \mathrm{pp}$

[46] Clowe D et al. A direct empirical proof of the existence of dark matter. Astrophysical Journal. 2006;648:L109-L113 